\title{
Regular, brief mindfulness meditation practice improves electrophysiological markers of attentional control
}

\author{
Adam Moore ${ }^{1}$, Thomas Gruber ${ }^{2}$, Jennifer Derose ${ }^{1}$ and Peter Malinowski ${ }^{1}{ }^{*}$ \\ School of Natural Sciences and Psychology, Liverpool John Moores University, Liverpool, UK \\ 2 Institute of Experimental Psychology I, University of Osnabrück, Osnabrück, Germany
}

\author{
Edited by: \\ Amishi P. Jha, University of Miami, \\ USA \\ Reviewed by: \\ Juliana Yordanova, Bulgarian \\ Academy of Sciences, Bulgaria \\ Lutz Jäncke, University of Zurich, \\ Switzerland \\ *Correspondence: \\ Peter Malinowski, School of Natural \\ Sciences and Psychology, Liverpool \\ John Moores University, Byrom \\ Street, Liverpool L3 3AF, UK. \\ e-mail: p.malinowski@ljmu.ac.uk
}

Mindfulness-based meditation practices involve various attentional skills, including the ability to sustain and focus ones attention. During a simple mindful breathing practice, sustained attention is required to maintain focus on the breath while cognitive control is required to detect mind wandering. We thus hypothesized that regular, brief mindfulness training would result in improvements in the self-regulation of attention and foster changes in neuronal activity related to attentional control. A longitudinal randomized control group EEG study was conducted. At baseline (T1), 40 meditation naïve participants were randomized into a wait list group and a meditation group, who received three hours mindfulness meditation training. Twenty-eight participants remained in the final analysis. At T1, after eight weeks (T2) and after 16 weeks (T3), all participants performed a computerized Stroop task (a measure of attentional control) while the 64-channel EEG was recorded. Between T1 and T3 the meditators were requested to meditate daily for 10 min. Event-related potential (ERP) analysis highlighted two between group effects that developed over the course of the 16-week mindfulness training. An early effect at left and right posterior sites 160-240 ms post-stimulus indicated that meditation practice improved the focusing of attentional resources. A second effect at central posterior sites $310-380 \mathrm{~ms}$ post-stimulus reflects that meditation practice reduced the recruitment of resources during object recognition processes, especially for incongruent stimuli. Scalp topographies and source analyses (Variable Resolution Electromagnetic Tomography, VARETA) indicate relevant changes in neural sources, pertaining to left medial and lateral occipitotemporal areas for the early effect and right lateral occipitotemporal and inferior temporal areas for the later effect. The results suggest that mindfulness meditation may alter the efficiency of allocating cognitive resources, leading to improved self-regulation of attention.

Keywords: meditation, mindfulness, cognitive control, EEG, Stroop, interference, attention

\section{INTRODUCTION}

During the last decade the scientific interest in the effects of meditation and mindfulness practice has experienced an unprecedented surge. A growing number of studies are confirming benefits of mindfulness practices in a broad range of psychologically relevant domains (Grossman et al., 2004; Chiesa and Serretti, 2009, 2011). After an initial phase of demonstrating general benefits, research is increasingly zooming in on more detailed questions regarding the underlying mechanisms that contribute to the observed changes.

Mindfulness meditation practices are considered to entail at least two central components: the training of attentional skills and the development of an equanimous, non-judgmental attitude toward one's own experiences, toward sensations, thoughts and feelings, where arising experiences are acknowledged without elaboration or reaction (e.g., Kabat-Zinn, 1990, 2003; Bishop et al., 2004; Malinowski, 2008). Although conceptualizations may differ in some specific details, as for instance the inclusion of additional components such as the intention to practice (Shapiro et al., 2006), or the provision of a more fine-grained classification of contributing factors (Dorjee, 2010), the development of attentional skills is included as a fundamental factor throughout (Lutz et al., 2008). The basic training of attentional skills is thought to underpin other changes that lead to positive health outcomes and well-being (Chiesa and Malinowski, 2011; Malinowski, 2012). Most importantly, attentional stability, clarity, and flexibility are thought to be prerequisites for maintaining a non-judgmental attitude toward one's experiences. In a first approximation these two components of mindfulness practice have been described in cognitive terms as focused attention and open monitoring (Lutz et al., 2008), which, depending on the particular meditation system, may be practiced selectively or in a combined fashion.

As the development and refinement of attentional skills appears fundamental to all forms of mindfulness meditation practice, it is not surprising that a major line of investigation focuses on revealing how meditation practice influences various aspects of attentional performance and the underlying brain mechanisms (e.g., Valentine and Sweet, 1999; Lutz et al., 2004; Wenk-Sormaz, 2005; Anderson et al., 2007; Brefczynski-Lewis et al., 2007; Jha et al., 2007; Chambers et al., 2008; van Leeuwen et al., 2009; Hodgins and Adair, 2010; van den Hurk et al., 
2010; Zeidan et al., 2010). Chiesa et al. (2011) provide a systematic review of studies into this topic that were published until May 2010. So far, the findings suggest that meditation practice may increase the efficiency of attention networks (Corbetta and Shulman, 2002; Raz and Buhle, 2006; Posner and Rothbart, 2007), where executive control functions that comprise of mental set shifting, the updating and monitoring of information and, crucially, the inhibition of prepotent responses play a central role (Miyake et al., 2000).

However, extant research varies greatly regarding study design, levels of meditation experience, and various other aspects, meaning that straightforward conclusions regarding possible causation are difficult. For instance, the majority of studies only used a cross-sectional approach, which does not answer the question whether meditation practice is causally involved in observed differences between meditators and non-meditators. Other studies investigated meditators with rather varied meditation experience or from different meditation traditions, and a number of other studies employ intervention packages like the mindfulness-based stress reduction program (MBSR; Kabat-Zinn et al., 1985, 1987, 1992), which entails other aspects like yoga exercises and psychoeducative components. While all of these studies are worthwhile and make important contributions to our understanding as to how mindfulness practice influences attentional functions (Williams, 2010), it is important to complement these findings with studies that directly investigate the effects of meditation practice over time, while keeping additional aspects that may influence the results to a minimum.

In studies that focused on investigating a specific meditation practice rather than employing more comprehensive intervention programs like MBSR the time period and the amount of daily meditation practices varied considerably. At the lower end are studies that used only very brief periods, as for instance Polak (2009), who investigated the effect of only two $15 \mathrm{~min}$ meditation sessions or a study by Wenk-Sormaz (2005), where participants completed three times $20 \mathrm{~min}$ of meditation practice. At the upper end changes resulting from meditation retreats, where participants are withdrawn from ordinary life for longer periods of time, were investigated. Chambers and co-workers (2008) investigated the effects of a 10-day meditation retreat, Jha et al. (2007) and van Vugt and Jha (2011) studied the effects of one-month mindfulness meditation retreats while other studies investigated the effects of different three month meditation retreats (Slagter et al., 2007; Lutz et al., 2009; MacLean et al., 2010; Jacobs et al., 2011; Sahdra et al., 2011). Between these endpoints a few further studies used dosages of meditation practice that can more easily be integrated into ones daily routines. In a study by Semple (2010) participants were asked to practice mindfulness meditation for $20 \mathrm{~min}$ twice per day over a period of one month. Tang et al. (2009) employed 20 min of daily integrative mind-body training (IBMT) over a period of five days and another study by Tang et al. (2010) asked participants to practice IBMT for a period of one month, for $30 \mathrm{~min}$ daily, five days per week. As this brief overview shows, there is little coherence regarding the amount or dosage of meditation practice. It is thus difficult, if not impossible, to find any guidance regarding the "right" amount and duration of practice. As has been pointed out recently, this issue has not yet been addressed systematically (Slagter et al., 2011).

The present study was designed to address our primary interest of investigating the effects of meditation practice that can easily be integrated into one's life, without requiring major changes in daily routines or life style. A related secondary aim was to study lower boundaries of meditation practice. We were curious to find out whether a rather modest dosage would yield any benefits in terms of cognitive processing. It was thought that $10-15 \mathrm{~min}$ of daily meditation practice would be a minimum time period allowing participants to settle in the meditation practice and develop some attentional stability. An additional question was what time period would be required for any changes to appear. Due to the low daily meditation dose, we considered that practice effects might require longer time to emerge. Balancing resources, the required commitment of participants and avoiding interference through breaks due to summer vacations, we settled for a total meditation period of 16 weeks. To get some indication regarding the time course of the changes, an intermediate testing session was included halfway through the study, after eight weeks. To reduce the possible influence of group dynamics that would make unequivocal interpretations of our results more difficult, we furthermore opted for an approach that includes only $3 \mathrm{~h}$ of group contact time in groups of three to six participants, early on in the study.

In line with our aim of investigating elementary aspects of mindfulness meditation, a meditation practice was chosen that is common to many forms of mindfulness training. For instance, the mindful breathing practice that was employed here is an integral part of MBSR (Kabat-Zinn, 1990) and MBCT (Segal et al., 2002), is the starting point in contemporary meditation programs as for instance the shamatha training composed by Alan Wallace or of mindfulness practice as explained by Gunaratana (1992). At the same time it is a basic component of different traditional buddhist meditation systems, ranging from early buddhist sources like the Anāpānasati Sutta or the Satipatthāna Sutta (Bhikkhu Bodhi, 1995) to classical Tibetan buddhist instructions (Karmapa Wangchug Dorje, 2009). Thus, the mindful breathing exercises used in this study bear relevance to a large variety of mindfulness approaches and practices.

As we were particularly interested in the effects of meditation practice on executive functions, we employed the Stroop Word-Color Task (Stroop, 1935; MacLeod, 1991), which in a previous study in our lab has revealed large differences between meditators and non-meditators (Moore and Malinowski, 2009). Central to the Stroop task is that the automatized reading of words leads to performance decrements if the semantics of a color word conflicts with naming/indicating the color this word is printed in (e.g., "BLUE" presented in red). Good performance on this task would be indicative of good cognitive control and relatively low automaticity or impulsivity of one's responses. Because the actual meditation training is very different to the Stroop task itself, improvements in the Stroop task would be of interest regarding the question, whether abilities trained in meditation generalize to other tasks and domains beyond the training itself. Thus, although the training consists of merely directing and redirecting one's attention to breathing-related sensations 
and to disengage from or non-engage with arising thoughts and emotions, changes in the automaticity of reading that is part and parcel of the congruency effect in the Stroop task would be remarkable. As skill learning is usually very task-specific and does not easily generalize beyond the specific tasks, stimuli, or contents (Green and Bavelier, 2008), such changes would furthermore highlight the possibility that mindfulness practice leads to changes of underlying processes rather than specific content (Slagter et al., 2011). Furthermore, such changes could be an indication of improved impulse control or even a fundamental change as to how individuals relate to their experiences, possibly having relevance beyond the cognitive domain itself (Chambers et al., 2009).

To get a precise estimation as to how meditation practice may change the involved neuronal processes, we employed 64-channel EEG recordings, while the participants engaged in a computerized version of the Stroop task. We hypothesized that, compared to a non-meditating, wait list control group, engagement in a regular, brief meditation practice would lead to improvements in attentional performance as indexed by the behavioral Stroop interference effect, which would also be reflected in changes in several electrophysiological parameters. As previous studies found that this Stroop effect is reflected in a late negativity (LN) that typically starts around 350-400 ms after stimulus onset, we expected to find meditation-related changes in this event-related potential (ERP) component (e.g., Liotti et al., 2000; Hanslmayr et al., 2008). First electrophysiological investigations of attention effects in mindfulness meditators furthermore report a reduction of a slightly earlier positivity — the P3 component-in response to a distracter sound (e.g., Cahn and Polich, 2009) and as an indicator of improved resource allocation in the attentional blink task (Slagter et al., 2007), which requires the temporal allocation of selective attention. Accordingly, we also considered this component. As for some other types of meditation also changes in a negative deflection occurring before the P3 in a time range starting from around $150 \mathrm{~ms}$ after stimulus onset were reported (Cahn and Polich, 2006), and this earlier negative component (N2) has been implicated in attentional processes (Folstein and van Petten, 2008), it was considered as well. As both the $\mathrm{N} 2$ and the $\mathrm{P} 3$ have been shown to reflect attentional control mechanisms, while the LN is considered to be an indicator of the Stroop interference effect, we expected that mindfulness practice would influence some or all of these ERP components.

To sum up, the aim of the current research was to investigate whether a simple, brief meditation practice carried out regularly for 16 weeks will lead to detectable changes in cognitive performance and associated neural processes. To reduce the possible influence of some of the factors that made unequivocal interpretations of previous results difficult, we opted for an approach that includes only a minimum of group contact time $(3 \mathrm{~h})$ and a limited amount of daily meditation practice $(10 \mathrm{~min})$, thus allowing participants to carry on with their daily routines without much change or disruption. Furthermore, this "ten-minutes-per-day" approach that we employed may be a more viable option, for people who may consider integrating mindfulness practice into daily life.

\section{METHODS}

\section{PARTICIPANTS}

Forty healthy adults (13 males; mean age 35.4 years) were recruited via a combination of online advertisements and from a psychology participant panel maintained at Liverpool John Moores University (LJMU). To be included in the study participants had to be meditation naïve (no previous meditation experience), have normal or corrected-to-normal visual acuity, confirm they have no ongoing or recent mental health problems or neurological disorders (e.g., epilepsy) and confirm they are not receiving any psychopharmacological treatments. Thirty-eight participants described themselves as "White" or "White/British," one as "White/Irish" and one as "White/Caribbean." Fifteen participants classed their religious background as Christian (Christian, Roman Catholic, Church of England), one as Atheist, one as Agnostic. The remaining participants stated no religion. Three students took part in the study. Most of the participants were in full-time or part-time employment or in voluntary work. The majority of participants were educated at least to undergraduate level, with 11 participants with postgraduate qualifications. Due to the nature of the design the participants were aware of the general aims of the study, but no specific hypotheses were explained to them.

The study was carried out in line with the ethics guidelines of the British Psychological Society and was approved by the LJMU Research Ethics Committee. All participants provided written,

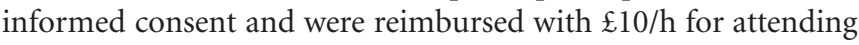
the six testing sessions.

Participants were randomly allocated to the meditation group or the waitlist control group, with the restriction that age and gender composition were matched across groups. Figure 1 summarizes the flow of participants through the study. Twelve participants in the meditation group and 16 in the control group were included in the final analysis of the EEG data. As far as could be ascertained, drop-outs in the meditation group were motivated by personal or health reasons not related to the study itself.

Initial tests of baseline (Time 1) measures are presented in Table 1 and confirm that the two groups did not differ significantly with respect to age, gender, the different self report measures, or performance measures on the Stroop task.

\section{SELF-REPORT MEASURES}

\section{Global well-being}

The Subjective Happiness Scale (SHS, Lyubomirsky and Lepper, 1999) was used to assess the global, subjective assessment of participants' own happiness and well-being. The SHS is a brief four-item questionnaire scored on a seven-point Likert scale and includes items like "In general I consider myself a very happy person." High total scores reflect high levels of global well-being/happiness. The SHS has been successfully used in different community-based and college-student samples, showing Cronbach's alpha values between 0.79 and 0.94 (Lyubomirsky and Tucker, 1998; Lyubomirsky and Lepper, 1999).

\section{Mindfulness}

The Five Facet Mindfulness Questionnaire (FFMQ) was used to assess different aspects of mindfulness that were expected to be 


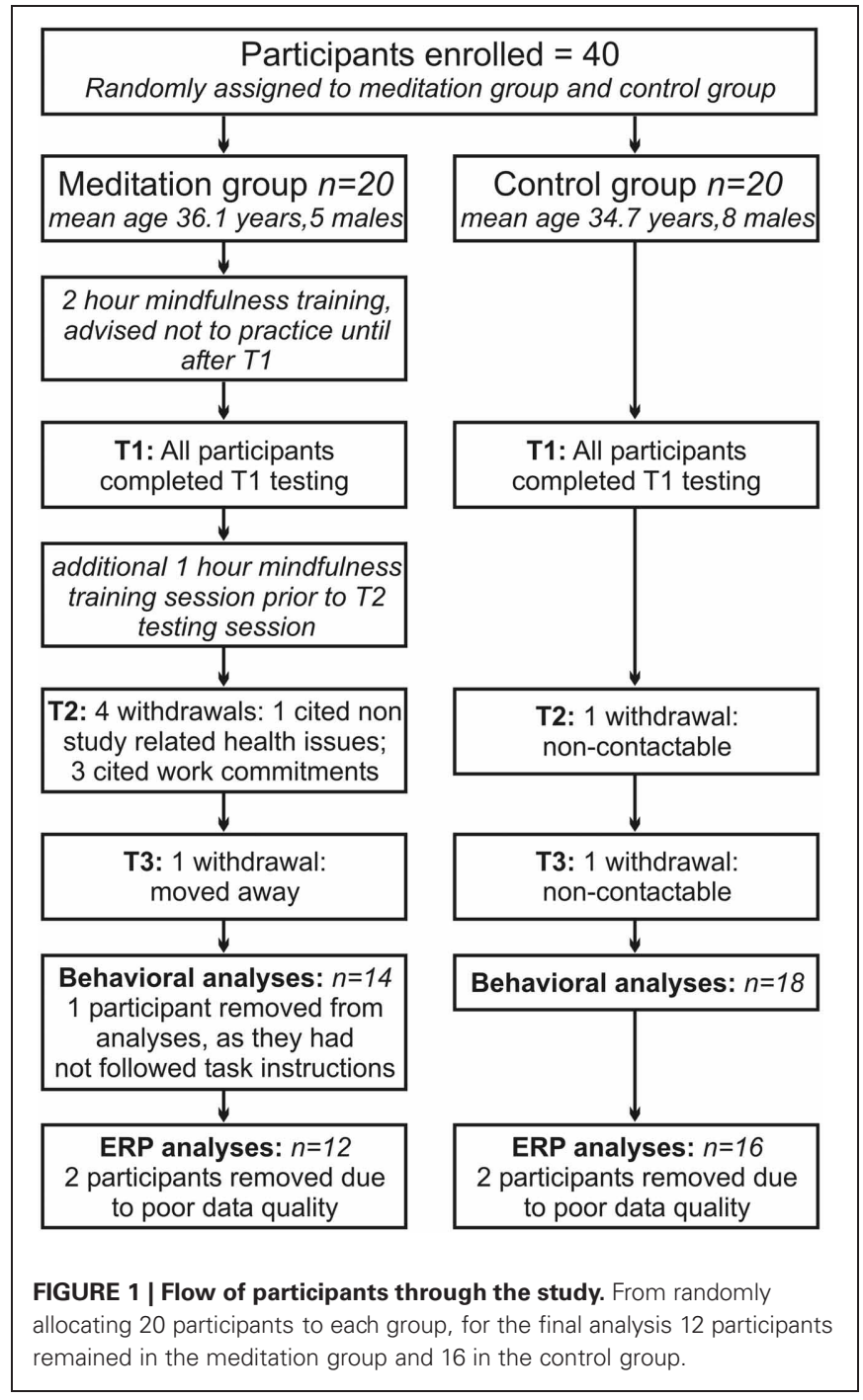

influenced by mindfulness practice. This 39-item-questionnaire was derived from an exploratory factor analysis of six existing selfreport measures of dispositional mindfulness (Baer et al., 2006). Validation on two samples (Baer et al., 2006, 2008) suggests a five factor structure: (1) Non-reactivity to inner experience (FFMQNR; seven items), e.g., "I watch my feelings without getting lost in them"; (2) Observing internal and external sensations including thoughts, emotions, sights, sounds, and smells (FFMQ-O; eight items) e.g., "I intentionally stay aware of my feelings"; (3) Acting with awareness describes attending to one's actions in the present moment and can be contrasted with automatic, impulsive, or habitual behaving (FFMQ-A; eight items), e.g., "It seems I am running on automatic without much awareness of what I'm doing"; (4) Describing involves labeling internal experiences with words (FFMQ-D; eight items), e.g., "When I have a sensation in my body, it's hard for me to describe it because I can't find the right words"; (5) Non-judging of experience means refraining from value judgments or self-criticism (FFMQ-NJ; eight items) "I tend to evaluate whether my perceptions are right or wrong." The response format comprises a five-point Likert scale $(1=$ never or very rarely true, rarely true, sometimes true, often true, and $5=$ very often or always true). After reversing the scores for the 19 negatively worded items, scores between 1 and 5 are summed to produce totals for each subscale and a total scale score (range: 39-195). The FFMQ has been shown to have good internal consistency and significant relationships in the predicted directions with a variety of constructs related to mindfulness. The internal consistencies (Cronbach $\alpha$ ) for these facets have been reported as 0.75 for FFMQ-NR, 0.83 for FFMQ-O, 0.87 for FFMQ-A, 0.91 for FFMQ-D, and 0.87 for FFMQ-NJ (Baer et al., 2006).

\section{Meditation log}

On a weekly basis participants in the meditation group completed a brief meditation diary (online or paper-pencil version), which recorded how often they meditated in a given week and the average length of the meditation sessions.

\section{PROCEDURES}

Potential participants received detailed information regarding the study, completed a screening questionnaire, signed a consent form, and were then randomly allocated to the meditation or control group.

Over the course of approximately 16 weeks, participants were tested at three time points (T1, T2, T3; 8-10 weeks apart). At each time point participants first completed the self-report questionnaires and then performed the experimental task, while the EEG was recorded. Two testing sessions of approximately $90 \mathrm{~min}$ length were conducted at each time point, as several other tests were carried out that are not reported in this paper. Around $\mathrm{T} 1$, the meditation group received introductory $2 \mathrm{~h}$ mindfulness training, in groups of three to six participants. In order to obtain accurate baseline data the meditators were instructed not to begin practicing meditation until after their first testing sessions. A follow up $1 \mathrm{~h}$ meditation training session was given to them prior to $\mathrm{T} 2$ and throughout the study the participants were able to contact the meditation teacher to answer questions or give further instruction.

\section{MEDITATION INSTRUCTION}

Participants in the meditation group were introduced to a simple mindful breathing meditation by a meditation teacher with more than 15 years of teaching experience. In this meditation the meditator is required to focus their attention on the sensations accompanying their breathing, either attending to the experience at the nostrils, around the diaphragm or the movement of the abdomen when in- and exhaling, without manipulating the breath in any form. Whenever the attention would slip or wander off, the task would be to become aware of it and, without further elaboration, to redirect the focus of attention to the sensation of breathing. In addition to this focusing of attention, participants were instructed to observe other mental experiences, arising thoughts, feelings or sensation, trying not to judge or evaluate them, and maintain a curious, non-elaborating attitude toward them. This meditation instruction is in line with common psychological mindfulness conceptualizations that emphasize the development of attentional abilities combined with a specific, non-evaluative attitude toward the different mental experiences that may arise (e.g., Bishop, 2002; 
Table 1 | Summary of tests for baseline differences, with mean values, standard deviations (in brackets), and respective statistical values for the comparison between meditation group and control group.

\begin{tabular}{llll}
\hline & Meditation group & Control group & Statistical values \\
\hline Age (years) & $36.9(12.1)$ & $34.6(11.4)$ & $t(26)=0.53, p=0.60$ \\
FFMQ-total & $126.9(15.6)$ & $136.8(12.6)$ & $t(26)=-1.86, p=0.08$ \\
SHS & $22.0(2.9)$ & $21.3(3.4)$ & $t(26)=0.62, p=0.54$ \\
RT all trials (ms) & $789.3(102.7)$ & $738.6(127.9)$ & $t(26)=1.13, p=0.27$ \\
RT congruent (ms) & $741.0(102.5)$ & $683.3(117.6)$ & $t(26)=1.35, p=0.19$ \\
RT incongruent (ms) & $840.2(110.7)$ & $798.0(148.9)$ & $t(26)=0.82, p=0.42$ \\
Accuracy all trials (\%) & $95.9(2.9)$ & $95.6(4.7)$ & $t(26)=0.23, p=0.82$ \\
Accuracy congruent (\%) & $98.0(2.4)$ & $98.0(2.8)$ & $t(26)=-0.14, p=0.89$ \\
Accuracy incongruent (\%) & $94.0(4.1)$ & $93.0(7.6)$ & $t(26)=0.41, p=0.68$ \\
RT variance all trials (ms) & $186.2(33.4)$ & $181.6(46.2)$ & $t(26)=0.29, p=0.77$ \\
RT variance congruent (ms) & $169.4(35.7)$ & $159.5(51.6)$ & $t(26)=0.57, p=0.57$ \\
RT variance incongruent (ms) & $185.7(37.1)$ & $179.9(43.5)$ & $t(26)=0.37, p=0.71$ \\
Inverse efficiency all trials & $8.3(1.3)$ & $7.8(1.7)$ & $t(26)=0.77, p=0.45$ \\
Inverse efficiency congruent & $7.6(1.2)$ & $8.0(1.4)$ & $t(26)=1.21, p=0.24$ \\
Inverse efficiency incongruent & $9.0(1.5)$ & $t(26)=0.30, p=0.77$ \\
\hline
\end{tabular}

Shapiro et al., 2006; Malinowski, 2008; Chiesa and Malinowski, 2011; Malinowski, 2012). For the period between T1 and T3 (16 weeks) participants were asked to meditate regularly for a minimum of $10 \mathrm{~min}$ per day, at least five days per week and to record frequency and duration in their meditation log on a weekly basis. The participants did not receive any particular instructions regarding the body posture beyond the emphasis of trying to sit in an upright, relaxed position with a straight back. They had the liberty to meditate on a chair, meditation stool, or cushion. Given the relatively small sample size and low dosage of meditation we did not expect the specific meditation posture to have a discernable effect and thus did not record these details.

\section{TASK DESIGN AND STIMULI}

Stimuli in the Stroop task were the four color words RED, BLUE, GREEN, and YELLOW, presented in the same color as the written word in congruent trials (e.g., RED presented in red) and in different colors (e.g., RED presented blue) in incongruent trials. The task was presented on a 21 -inch CRT-monitor $(100 \mathrm{~Hz}$ vertical refresh rate, $1024 \times 768$ resolution) and was controlled by the Cogent 2000 toolbox (v1.25) running in the Matlab environment (Mathworks, http://www.mathworks.com). Words were presented in the Arial Font (font size $48 \mathrm{pt}$ ), viewed at a distance of approximately $90 \mathrm{~cm}$. Each incongruent stimulus appeared in each of the three other colors with equal frequency. Participants were instructed to respond as fast and accurately as possible and to indicate the color each word was presented in, while ignoring the semantic meaning of the word. Four keys on a standard QWERTY keyboard were used to enter their responses, using the keys "a" (red, left middle finger), "." (blue, left index finger), "x" (green, right index finger), and "' " (yellow, right middle finger). The keys were color-coded and chosen to provide optimum comfort for the participant whilst responding.

At the beginning of each trial a fixation cross was presented for $500 \mathrm{~ms}$, followed by the color word, which remained on the screen for $1500 \mathrm{~ms}$. The trial concluded with a variable inter-trial interval of between 850 and $1100 \mathrm{~ms}$. The stimulus always appeared centrally on the screen, replacing the fixation cross. The experiment began with a color-to-key acquisition phase which consisted of 48 trials that were similar to those used in the experimental blocks. During this phase, mistakes were highlighted by an audible tone and accuracy and reaction time feedback was given following completion of this phase. The experimental phase consisted of three blocks of 48 trials (50\% congruent, 50\% incongruent trials) for a total of 144 trials and 72 trials per condition. Each trial block lasted approximately $3 \mathrm{~min}$ and was followed by a $20 \mathrm{~s}$ break before the subsequent block.

\section{ELECTROPHYSIOLOGICAL RECORDINGS}

EEG was recorded continuously from $64 \mathrm{Ag} / \mathrm{AgCl}$ electrodes with a BioSemi Active-Two amplifier system (BioSemi, Amsterdam, Netherlands). For monitoring eye movements and blinks the horizontal and vertical electrooculogram (EOG) was recorded with supra- and infraorbital electrodes on the left eye and two electrodes placed next to the external canthi. EEG and EOG were sampled at $512 \mathrm{~Hz}$. Two additional electrodes (Common Mode Sense [CMS] and Driven Right Leg [DRL]) were used as reference and ground (see www.biosemi.com/faq/cms\&drl.htm for details). For further off-line analysis, the average reference was used.

EEG was segmented to obtain epochs starting $200 \mathrm{~ms}$ prior and $800 \mathrm{~ms}$ following stimulus onset. Pre-processing of data was performed in EEGLAB version 9.03 (Delorme and Makeig, 2004). The Fully Automated Statistical Thresholding for EEG artifact Rejection procedure (FASTER, Nolan et al., 2010) was employed for removing artifacts from the data. Using a predefined $z$-score threshold of \pm 3 for each parameter, artifacts were detected and corrected regarding single channels, epochs, independent components (based on the infomax algorithm, Bell and Sejnowski, 1995) and single-channel single-epochs. Remaining artifactual independent components and epochs containing artifacts were removed after visual inspection. Data were filtered offline with a 
$1 \mathrm{~Hz}$ high pass filter. A pre-stimulus baseline from $-60 \mathrm{~ms}$ to $0 \mathrm{~ms}$ was applied.

No between group differences existed regarding the number of trials available for analysis (Meditation group: 129.0, 132.3, and 131.8 Control group: 131.2, 131.7, and 130.3 for T1, T2, and $\mathrm{T} 3$, respectively) or the amount of independent components that were removed from the data (Meditation group: 9.3, 10.2, and 8.8; Control group: 11.4, 8.8, and 9.3 for $\mathrm{T} 1, \mathrm{~T} 2$, and $\mathrm{T} 3$, respectively).

\section{DATA ANALYSIS}

\section{Analysis of behavioral and self-report data}

For analyzing the behavioral and self-report data we conducted mixed ANOVAs with Group $\times$ Time $\times$ Congruency as factors for response times (RTs), response variability, and response accuracy and Group × Time for the self-report measures. As an estimate for the variability of responses over time we used the standard deviation of the RTs of all correct trials in each condition. To account for possible criterion shifts or influences of speedaccuracy tradeoffs, we furthermore analyzed the inverse efficiency scores, derived by dividing the mean RT by the proportion of correct responses, calculated separately for each condition and each participant (Akhtar and Enns, 1989; Christie and Klein, 1995).

Whenever the sphericity assumption (equality of variances) had been violated (Mauchly's test), Greenhouse-Geisser estimates of sphericity were employed to adjust the respective degrees of freedom.

All analyses of behavioral and self-report data were carried out twice, once including all participants that completed the study (14 meditators, 18 controls) and once limited to those participants that were included in the final analysis of EEG data (12 meditators, 16 controls). For consistency we will subsequently only report the latter, because the pattern of relevant results was identical for both approaches.

\section{Analysis of event-related potentials (ERP) - electrode space}

A $16 \mathrm{~Hz}$ low pass filter was applied prior to all ERP analysis. Based on the grand mean evoked potential (see Figure 2), three ERP components of interest were defined: N2 (160-240 ms), P3 (310-380 ms) and a late negative deflection (LN; 400-600 ms) for incongruent stimuli, typical for the Stroop task (e.g., Liotti et al., 2000). Mean amplitudes averaged across the respective time window were calculated for the amplitude maxima identified in the scalp topographies of each component and were subjected to Group $\times$ Time $\times$ Congruency mixed ANOVAs. Of particular interest for this study were interaction effects that included the factors Group and Time, as they would indicate that the respective ERP amplitudes were influenced differentially by meditation practice. Accordingly, the analysis primarily focuses on these interactions. As an estimate of the strength of the effect we calculated the effect size $r$ for these interactions.

As for the behavioral and self-report data, whenever the assumption of variance equality had been violated we employed the Greenhouse-Geisser procedure to adjust the respective degrees of freedom.

\section{Analysis of event-related potentials (ERP) - source space}

To get a general indication of brain areas that may be selectively influenced by meditation practice, we applied Variable Resolution

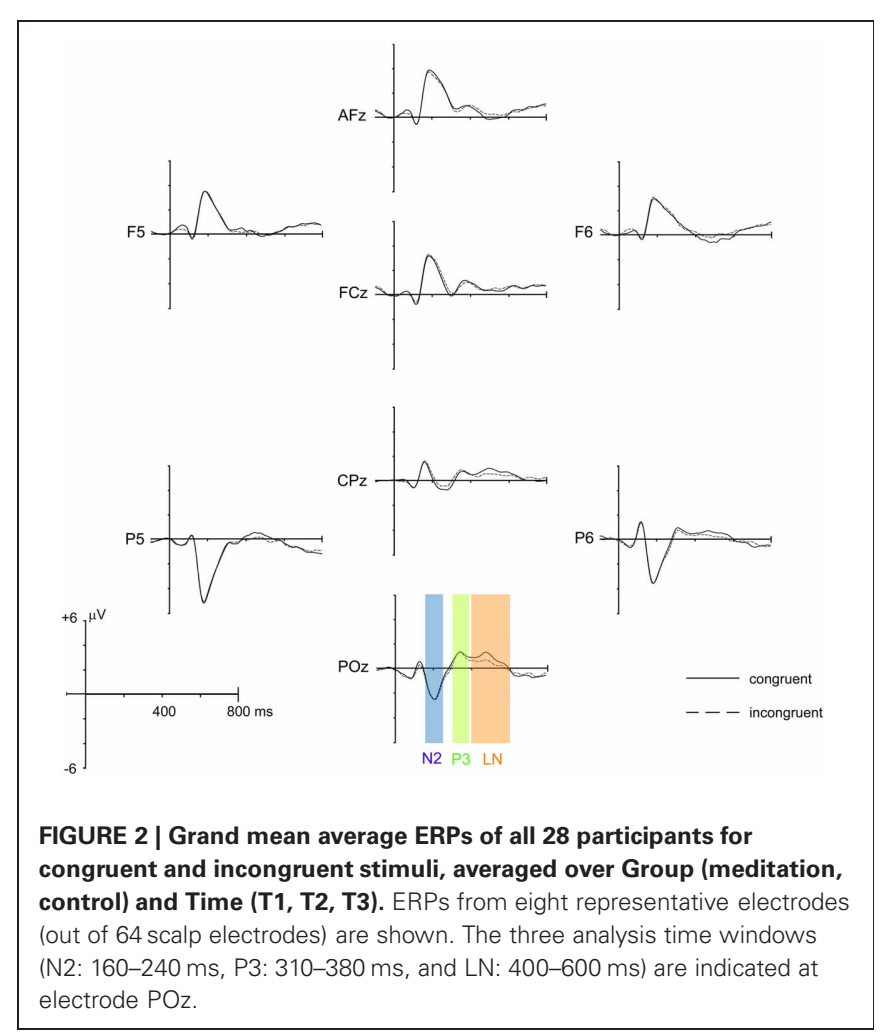

Electromagnetic Tomography (VARETA; Bosch-Bayard et al., 2001) to localize the cortical generators of the relevant ERP components that were identified in the electrode-space ERP analysis. This procedure was applied separately for each factorial combination of Group, Time, and Congruency. The VARETA approach provides the spatially smoothest intracranial distribution of current densities in source space which is most compatible with the amplitude distribution in electrode space (Gruber et al., 2006). The inverse solution consisted of 3244 grid points ("voxels") of a 3D-grid ( $7 \mathrm{~mm}$ grid spacing). This grid and the arrangement of 64 electrodes were placed in registration with the average probabilistic MRI brain atlas ("average brain") produced by the Montreal Neurological Institute (MNI; Evans et al., 1993). To localize the activation difference between $\mathrm{T} 1$ and $\mathrm{T} 3$ for each component and congruency condition, statistical comparisons were carried out by means of paired $t$-tests for the meditation group and control group. Activation threshold corrections accounting for spatial dependencies between voxels were calculated by means of false discovery rates (Benjamini and Hochberg, 1995). All statistical parametric maps were thresholded at a significance level of $p<0.001$.

\section{RESULTS}

\section{TEST FOR GROUP DIFFERENCES AT BASELINE}

Group $\times$ Congruency ANOVAs for RT, accuracy, RT-variability, and inverse efficiencies did not yield any significant main effects for Group nor significant Group $\times$ Congruency interactions (all $p>0.28$ ) at T1. As summarized in Table 1, no significant differences between meditation group and control group were present when direct comparisons at T1 ( $t$-tests) were calculated. 


\section{BEHAVIORAL DATA AND SELF-REPORT MEASURES Response times}

For RTs significant main effects of Time $[F(1.60,41.48)=4.953$, $p=0.018]$ and Congruency $[F(1,26)=110.554, p<0.001]$ were observed, indicating that overall the mean RTs decreased throughout the experiment (T1: $760 \mathrm{~ms}, \mathrm{~T} 2: 732 \mathrm{~ms}, \mathrm{~T} 3: 732 \mathrm{~ms}$ ) and that responses to congruent stimuli were faster $(689 \mathrm{~ms})$ than to incongruent stimuli $(797 \mathrm{~ms})$. There were no other significant effects. Importantly, neither the Group $\times$ Time interaction nor the Group $\times$ Time $\times$ Congruency interaction were significant ( $p=0.39$ and $p=0.15$, respectively).

\section{Response time variability}

For the variability of RTs a significant main effect of Congruency $[F(1,26)=43.609, p<0.001]$ was observed, reflecting that there was a lower RT variability for congruent $(\mathrm{SD}=154 \mathrm{~ms})$ than for incongruent $(\mathrm{SD}=181 \mathrm{~ms})$ conditions. Again, no effect that would reflect differential changes in meditation group and control group were present and the respective interaction effects were far from being significant (both $p>0.56$ ).

\section{Response accuracy}

Only the main effect of Congruency was significant $[F(1,26)=$ 33.604, $p<0.001$, congruent: $98.3 \%$, incongruent: $95.1 \%$ ] and no indication of differential changes between the groups emerged (both $p>0.26$ ).

\section{Inverse efficiency}

There were significant main effects of Time $[F(1,26)=4.408, p=$ $0.008]$ and of Congruency $[F(1,26)=85.224, p<0.001]$ but no further significant effects that would indicate differential changes between groups (both $p>0.14$ ).

Overall, the analyses of the behavioral results confirm that the task manipulation was effective, reflected by the influence of Congruency on task performance. Regarding possible training effects, only an initial speeding up of responses from T1 to T2 was observed, which is also reflected in improved efficiency scores. Beyond that, the data show that behavioral performance did not change differentially for meditation and control group and did not improve after T2.

\section{Mindfulness}

For the total mindfulness score, which combines the scores on the five FFMQ subscales, a significant main effect of Time $[F(1.640,42.645)=5.832, p=0.009]$ was observed, indicating that overall the mindfulness scores increased from T1 (132.6) to T3 (138.3). This effect was further qualified by a significant interaction between Group and Time $[F(1.640,42.645)=5.077$, $p=0.015]$. As Figure 3 shows, the increase of mindfulness from $\mathrm{T} 1$ to $\mathrm{T} 3$ is more pronounced in the meditation group (T3-T1: 11.8 points, $p=0.015$ ) than in the control group (T3-T1: 1.1 points, $p=0.650$ ). Although the figure appears to suggest a difference between meditation and control group at T1, testing for baseline differences (Table 1) showed that these differences were not significant $(p=0.08)$ and may just be an effect of random group allocation.

The analysis of the FFMQ subscales revealed a stronger increase in the meditation group than in the control group for

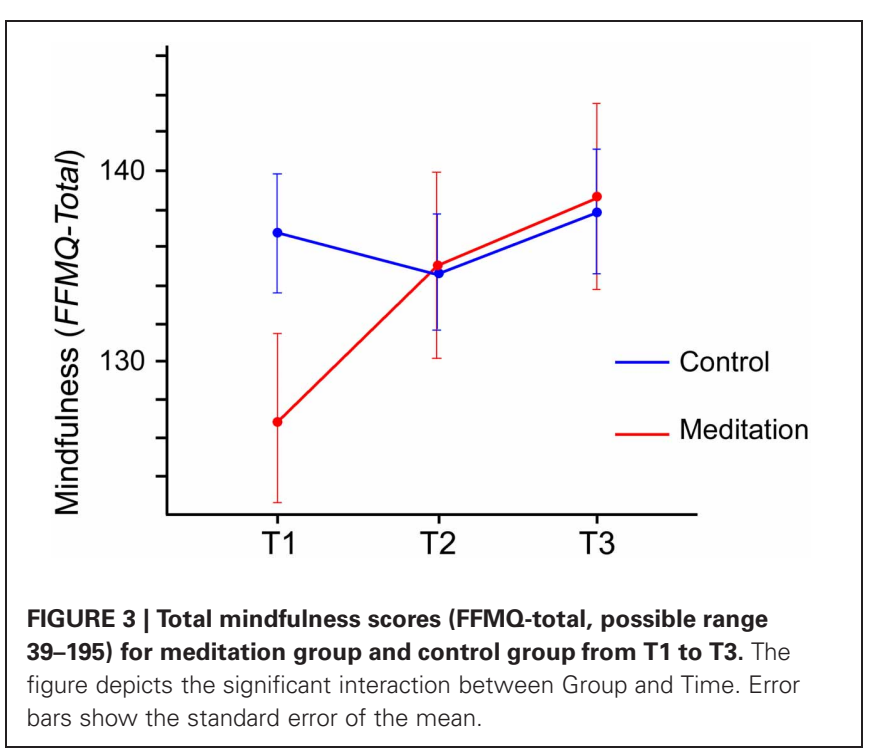

the observing (FFMQ-O) subscale $[F(2,52)=4.300, p=0.019]$ and the non-reacting (FFMQ-NR) subscale $[F(2,52)=3.771$, $p=0.030]$. No other significant effects emerged from the analysis of the FFMQ subscales.

\section{Meditation time}

In general, the participants in the meditation group managed to adhere to the required meditation schedule. Based on the meditation logs, the mean time spent meditating during each session was $11.3 \mathrm{~min}$ (range: $6.2-21.5 \mathrm{~min}$ ) and the average number of meditations per week was 5.0 sessions (range: $2.6-8.7$ ).

\section{Mindfulness and meditation time}

To analyze whether the amount of meditation practice would predict the increase in self-reported mindfulness, we calculated the Pearson correlation between the total time spent meditating over the 16 weeks and the changes in mindfulness scores from T1 to T3 in the meditation group. As Table 2 shows, increases in the total mindfulness score correlated highly with total meditation time. Similarly, changes in three of the five FFMQ subscales (observing, acting with awareness, non-judging) correlated significantly with the total meditation time. None of the other behavioral measures correlated with total meditation time.

Table 2 | Pearson coefficients for the correlations between total amount of time spent meditating between $\mathrm{T} 1$ and $\mathrm{T} 3$ and increase in mindfulness (FFMO scores) from $\mathrm{T} 1$ to $\mathrm{T} 3$.

Total meditation time

FFMO-total

$0.771(0.002)$

FFMQ-O

$0.592(0.021)$

FFMQ-A

$0.577(0.025)$

FFMQ-D

$0.009(0.489)$

FFMQ-NJ

$0.805(0.001)$

FFMQ-NR

Values in brackets indicate one-tailed significance levels $(N=12)$. 


\section{ERP DATA}

\section{Plausibility check}

Before investigating the specific effects of meditation training and related between group differences, we confirmed that the resulting ERPs are in line with the typical patterns of electrical activity observed for the Stroop paradigm. Figure 2 depicts the grand mean ERPs averaged over Group (meditation, control) and Time (T1, T2, T3) of eight representative electrodes distributed over the whole scalp. The ERP deflections resemble the pattern usually found with the Stroop paradigm. Also the typical late negative deflection (LN) for incongruent trials, considered to be a robust reflection of the Stroop effect, is present in the data (e.g., Liotti et al., 2000).

\section{N2 component}

Maxima of the N2 were best captured by small clusters of left occipito-parietal (PO7, PO3, O1) and right occipitoparietal electrodes $(\mathrm{PO} 8, \mathrm{PO} 4, \mathrm{O} 2)$. Group $\times$ Time $\times$ Congruency ANOVAs revealed significant Group $\times$ Time interaction for the left $[F(2,52)=3.862, p=0.027, r=0.263]$ and for the right $[F(2,52)=4.273, p=0.019, r=0.276]$ electrode cluster. Planned Group $\times$ Time contrasts indicated that this effect reflects relatively higher amplitudes at T3 in the meditation group than in the control group. The relative increase of the $\mathrm{N} 2$ amplitude from $\mathrm{T} 1$ to $\mathrm{T} 3$ in the meditation group contrasted with an amplitude decrease in the control group for left and right clusters $[F(1,26)=$ $6.421, p=0.018, r=0.445$ and $F(1,26)=4.987, p=0.034, r=$ 0.401]. Figure 4(A) shows the changes in the ERPs of the two electrode clusters from T1 to T3. The grand mean spherical-spline interpolated T3-T1 topographical difference maps in Figure 4(C) show that the N2 amplitudes at left and right posterior sites tend to develop in opposite directions for meditation and control group. Figure 4(D) depicts the neuronal sources where the differences from $\mathrm{T} 1$ to $\mathrm{T} 3$ developed. In the meditation group significant decreases in source strength (salmon-colored) from T1 to T3 for congruent stimuli were observed in the left middle and superior frontal gyri, the left medial and lateral occipitotemporal gyri, and the left middle temporal gyrus. In comparison, an increase of source strength (green color) in the left medial and lateral occipitotemporal gyri was observed in the meditation group. For incongruent stimuli, the control group showed a decrease in source strength in the left lateral occipitotemporal and left inferior temporal gyri, whereas no significant changes were present in the meditation group.

\section{P3 component}

The analysis of the P3 component focused on the central posterior amplitude maximum at electrode $\mathrm{Pz}$. There was a significant Group $\times$ Time $\times$ Congruency interaction $[F(2,52)=4.711, p=$ $0.013, r=0.288]$. Planned contrasts revealed that this interaction was due to a relative decrease in the P3 amplitude in the meditators in the incongruent condition from $\mathrm{T} 1$ to $\mathrm{T} 3 \mathrm{com}-$ pared to the control group, which exhibited an amplitude increase $[F(1,26)=9.267, p=0.005, r=0.513]$. The ERPs presented in Figure 5(A) show these differential changes from T1 to T3. The grand mean spherical-spline interpolated T3-T1 difference maps in Figure 5(C) show the topographical distribution of the changes over time, with a maximum decrease over central posterior sites in the meditation group for incongruent trials, contrasted by an increase in the control group. Figure 5(D) provides an indication of the brain areas that show differential source strength at T1 and T3. For the congruent condition a slight decrease in left superior and middle temporal gyri was present that was not present in the meditation group. An important contrast appeared for the incongruent condition. Whereas an increase of source strengths was observed for the control group (left medial and lateral occipitotemporal gyri, left inferior temporal gyrus, and right lateral occipitotemporal gyrus) an opposing pattern appeared for the meditation group. Here the right lateral occipitotemporal gyrus and the right inferior temporal gyrus showed a decrease in source strength from $\mathrm{T} 1$ to $\mathrm{T} 3$.

\section{Late negative component}

The broad negative deflection had a central posterior maximum that was best captured with an electrode cluster comprising of $\mathrm{Pz}$, $\mathrm{POz}, \mathrm{P} 1$, and $\mathrm{P} 2$. The only significant effect that emerged from the analysis was a main effect of Congruency $[F(1,26)=8.219, p=$ $0.008]$, confirming the typical Stroop interference effect, with the ERP for incongruent stimuli being negatively deflected compared to the congruent condition (see Figure 2).

\section{DISCUSSION}

Sixteen weeks of regular, brief meditation practice significantly changed neuronal activity related to executive control functions in the Stroop task. These changes were, however, not accompanied by related improvements in behavioral performance and did not pertain to the late negative ERP component (400-600 ms) that typically reflects the behavioral interference effect in the Stroop task (e.g., Liotti et al., 2000; Hanslmayr et al., 2008).

Meditation practice led to a relative increase of lateral posterior N2 amplitudes over both hemispheres, irrespective of stimulus congruency. Estimation of the neural sources (VARETA) suggests that these changes in the meditation group were primarily driven by increased activity in the left medial and lateral occipitotemporal areas for congruent stimuli, contrasted by decreased activity in similar brain areas in the control group. These left-hemispheric areas of the ventral processing stream have previously been identified as being selectively involved in lexical tasks (e.g., Cohen et al., 2002; Cohen and Dehaene, 2004; Shaywitz et al., 2004), with a similar posterior N2 component as observed here (e.g., Adorni and Proverbio, 2009). It thus seems plausible that this effect reflects more successful or consistent attentional amplification, selective to the word stimuli that were used in this task. This interpretation is in line with the time course of enhanced stimulus processing when attending to non-spatial features of a stimulus. Typically, enhanced negative posterior ERP amplitudes appear from around 100 to $150 \mathrm{~ms}$ after stimulus onset (Hillyard and Anllo-Vento, 1998; Hillyard et al., 1998). Even more, the posterior N2 is particularly enlarged when attending to the color as compared to the form of a stimulus (Eimer, 1997). Thus, while the control group exhibited a habituation effect over the course of the study (and $3 \times 144$ trials), which was expressed in a reduction of the ERP amplitudes and the related cortical source strengths, the meditation group showed the opposite 


\section{N2 (160 - $240 \mathrm{~ms})$ at left posterior and right posterior electrode sites}

A
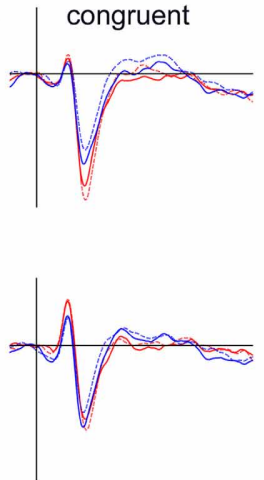

C<smiles>[CH]COC(=O)O</smiles>

$\mu \mathrm{V}$

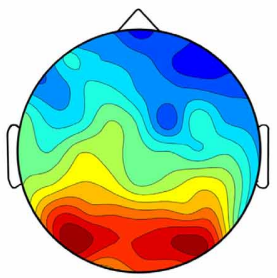

0

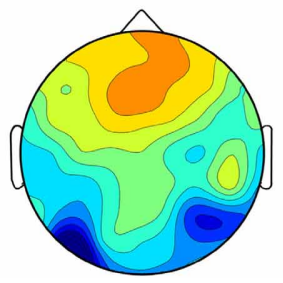

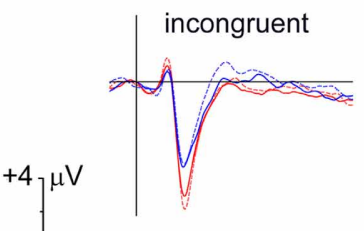

$400 \quad 800 \mathrm{~ms}$

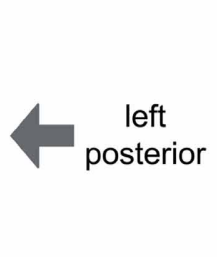

B

congruent

incongruent
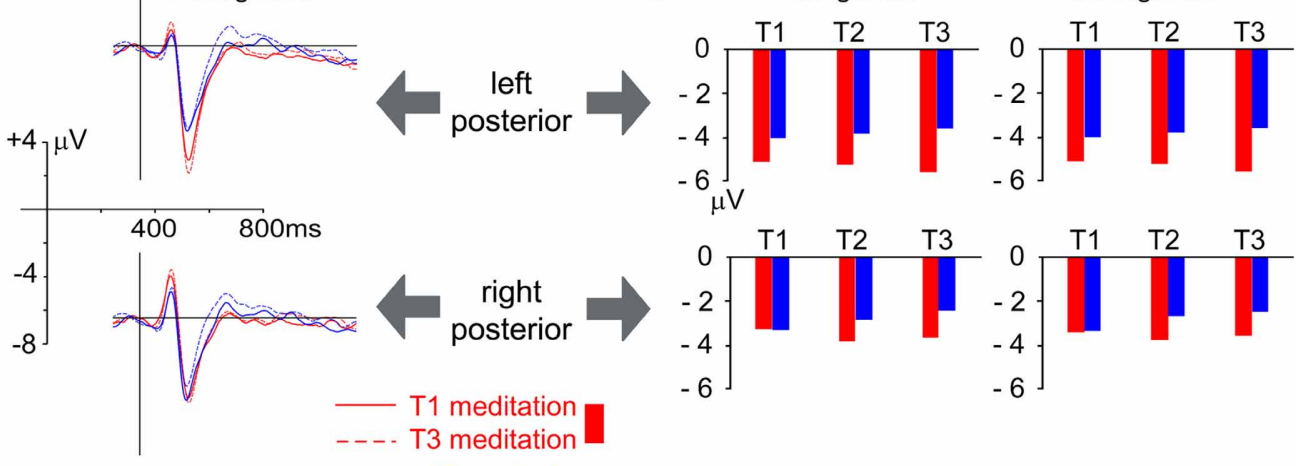

right
posterior
T1 meditation
-. T3 meditation
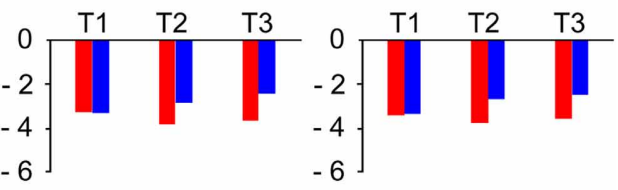

- T1 control

-... T3 control

D

congruent

incongruent
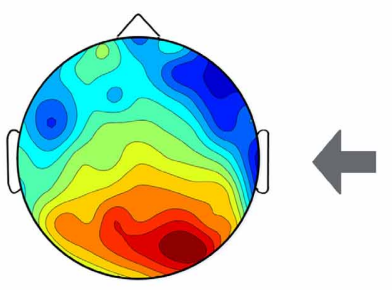

Control
Group
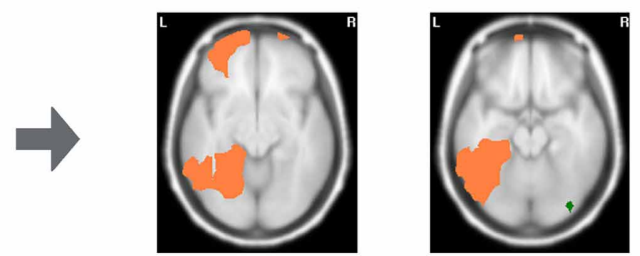

T. Meditation
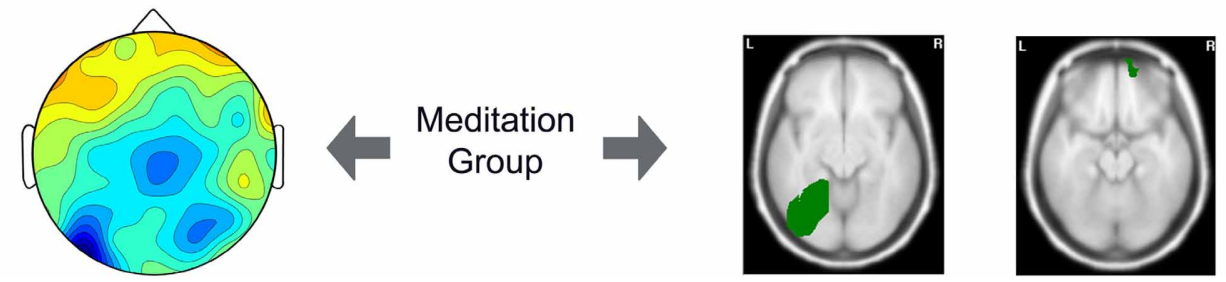

FIGURE 4 | Analysis of the N2 time window, from 160 to $\mathbf{2 4 0}$ ms. (A) Grand mean averages for meditation and control group for T1 and T3 averaged over left posterior electrodes (PO7, PO3, O1; upper row) and right posterior electrodes (PO8, PO4, O2; lower row). (B) Mean N2 amplitudes from T1 to T3 averaged over the same electrode clusters. (C) Spherical spline interpolated scalp topographies of the difference between $\mathrm{T} 1$ and $\mathrm{T} 3$ (T3-T1) for meditation and control group, separated for congruent and incongruent stimuli. Positive values indicate a decrease

in amplitudes; negative values indicate an increase in amplitudes. (D) Activation differences between T1 and T3 for each group and congruency, based on the localization of cortical generators with VARETA. Significant differences (threshold $p<0.001$ ) are presented for axial MNI slices at $Z=-10$ for congruent stimuli and at $Z=-17$ for incongruent stimuli (centers of gravity of the activation). Salmon-colored areas indicate a decrease in activation and green areas indicate an increase in activation.

pattern, where increased activation of task relevant cortical areas developed with meditation practice.

The second difference between meditators and controls was observed in the P3 component $(310-380 \mathrm{~ms})$. The majority of ERP studies of the Stroop task focus on later components starting around $400 \mathrm{~ms}$, as these tend to correlate with behavioral performance (Liotti et al., 2000), whereas the preceding P3 component appears to reflect earlier aspects of stimulus processing that, in themselves, are not the source of the behavioral Stroop interference effect (Ilan and Polich, 1999). Changes of the P3 over the course of the study were primarily observed for incongruent stimuli. While the participants in the control group exhibited an increase of the P3 amplitude for incongruent stimuli, a decrease was observed for the meditation group. The P3 decrease in electrode space was accompanied by significantly decreased signal strength in source space, which comprised lateral occipitotemporal and inferior temporal regions of the right hemisphere. These areas have been implicated in object recognition processes (Schendan and Kutas, 2002; Schendan and Stern, 2007). In addition, the temporal/parietal P3 component is considered to reflect attentional resource activation that is generated when perceptual stimulus discrimination occurs and is linked to related inhibition processes that are required when conflicting stimulus information is present (Polich, 2007). The pattern of results emerging for the P3 component thus suggests that through meditation practice the perceptual processing of incongruent stimuli becomes less resource demanding.

These findings bear similarities to the results from a previous study, where experienced meditators showed a reduced P3 amplitude to a distracter tone during an auditory oddball stimulation while they were meditating (Cahn and Polich, 2009). There are however, noteworthy differences to our study. In Cahn 


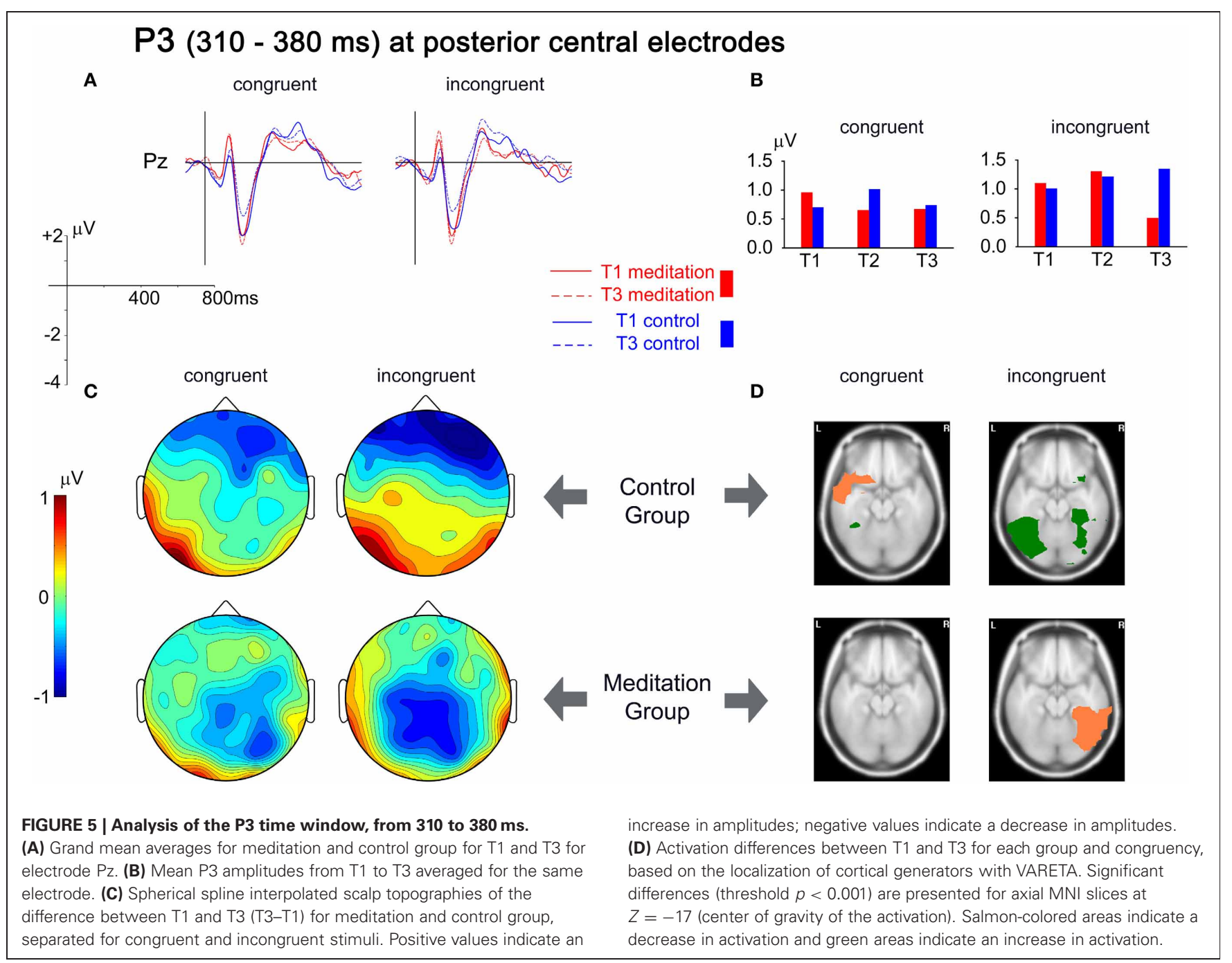

and Polich's study a meditation state was compared to a neutral thinking state, whereas we studied the effect of meditation in a task that was performed outside of the meditation practice. Furthermore, we investigated changes through meditation practice developing over time, while Cahn and Polich (2009) only tested at one time point and thus do not directly address the question of causal influences of meditation training. The parallels are nevertheless interesting, as they suggest that an ability that developed and is present during meditation practice appears to generalize to a different task performed when not meditating. It may indicate that state effects observed during meditation may translate into trait effects observed outside of meditation (Cahn and Polich, 2006), an assumption that underlies the idea that meditation practice generalizes into daily activities and extends to contexts separate from meditation practice (Hodgins and Adair, 2010; Slagter et al., 2011).

Furthermore, our results are in line with other studies suggesting that meditation practice leads to more effective brain resource allocation (Slagter et al., 2007, 2009). Slagter and co-workers employed the attentional blink paradigm to investigate how a three-month intensive meditation retreat changes the temporal deployment of attention compared to a non-meditating matched control group (Slagter et al., 2007, 2009). During the attentional blink task participants have to attend to a rapidly changing stream of stimuli (e.g., letters) and report the identity of two target stimuli (e.g., digits) after each trial. Performance to the second target in the stream is typically negatively affected if it appears within $500 \mathrm{~ms}$ after the first target, the so-called attentional blink effect (Shapiro et al., 1997). After the meditation retreat the meditators showed a reduced attentional blink effect. Furthermore, the P3b amplitude elicited by the first target stimulus was reduced in meditators after the retreat and the participants with the greatest decrease of the P3b amplitude also showed the largest decrease in attentional blink size (Slagter et al., 2007). Interestingly, the additional analysis of the phase of oscillatory theta activity following successfully detected second targets, showed a reduced cross-trial variability, considered to indicate that the deployment of attention was more consistent and that through meditation training attentional resources become more rapidly available to process additional information (Slagter et al., 2009).

The results from a recent fMRI study comparing meditators and matched controls on the Stroop task provide further 
support for our findings. Compared to a control group, meditators showed reduced activity in various brain areas subserving attention (Kozasa et al., 2012). The authors interpret their overall pattern of findings as evidence of enhanced efficiency in meditators that may result from improved sustained attention and impulse control.

When considering our results of enhanced N2 and decreased P3 amplitudes and source strengths in light of the reviewed findings, a possible interpretation emerges. We surmise that the more successful attentional amplification of the color word stimuli evidenced by increased N2 amplitudes/source strengths had the subsequent effect that fewer resources needed to be invested during object recognition processes, especially when incongruent stimulus information was processed, indexed by the decrease in P3 amplitudes/source strengths.

Confining the meditation training to a very simple, but fundamental, mindful breathing meditation, which often constitutes the first step into a more elaborate path of different meditation practices, gives confidence that the observed changes indeed stem from the meditation practice itself. Having kept the group sessions to a bare minimum (a total of $3 \mathrm{~h}$ ), makes it furthermore unlikely that unspecific group effects account for the changes. The fact that participants only meditated for very brief periods each day speaks against an explanation that life style changes could explain the observed differences, an influence that may well be relevant when studying the effects of longer daily meditation practices, of meditation retreats or when studying highly experienced meditators.

As meditation effects were compared to effects in a non-active waitlist control group, an alternative explanation might be that the observed effects merely result from the fact that the meditators were engaged in a novel regular activity per se, rather than being specific to the meditation practice. The current design cannot fully rule this out, but given that the observed effects are in line with results from several other studies into similar meditation practices, it appears likely that the effects are more specific. However, the general weakness of waitlist controlled designs in this respect needs to be acknowledged. The study tells us that engaging in $10 \mathrm{~min}$ of daily meditation practice for the given period has specific effects. It can, however, not be concluded that these effects are completely unique to meditation practice in general or to this specific type of mindfulness meditation in particular. While the mindfulness training had these effects, other practices or activities may have as well. Future studies will have to face up to the challenge of addressing the question how specific changes associated with meditation training actually are. Toward this end, control conditions that are matched with respect to somatic, mental, and cognitive demands but without actually being meditation practice will be required.

In this study the participants were required to record frequency and amount of meditation practice themselves. As the experimenters appeared to have a good rapport with the participants and it was emphasized that it is more important to provide accurate information than to fulfill a specific regime, we have no specific reason to doubt the honesty and accuracy of these records. We are, however, in no position to objectively confirm this. The fact that we found a positive relationship between mindfulness (FFMQ) and amount of meditation practice might be taken as a positive indicator, but as both are self-report measures they may be prone to similar distortions. Future studies may want to control actual meditation time more objectively. One needs to be aware, though, that this is only possible to a certain extent, because even if, for example, actigraphic measures of rest and activity cycles were available or sensors were integrated into meditation stools or cushions, we have to rely on participant reports whether during a period of physical rest they actually engaged in meditation practice.

An unexpected result of the study was that no differences in behavioral measures between meditation and control group appeared. This finding goes hand in hand with the lack of an effect of meditation practice on the LN, but is at odds with results from several other studies, which tended to show better performance of meditators over controls in similar measures of executive attention and conflict resolution (Chan and Woollacott, 2007; Jha et al., 2007; Moore and Malinowski, 2009). One important difference between such cross-sectional data and the study presented here is that a longitudinal design requires the repeated administration of the same experimental task. In the current study 144 trials of the Stroop task were administered at each time point (to a total of 432 trials). The fact that overall RTs did not improve after T2 (T2: $632 \mathrm{~ms}$, T3 $632 \mathrm{~ms}$ ) and that accuracy was above 95\% for incongruent trials, suggests that a performance ceiling might have been reached. A further difference to the cross-sectional study that showed the clearest performance differences between mindfulness meditators and a control group (Moore and Malinowski, 2009) was, that a verbal paper-pencil version of the Stroop task was used, whereas here a computerized version with manual button presses was employed. Several authors have highlighted that the way of administering the Stroop task has an influence on behavioral results and the interference effects in particular (Kindt et al., 1996; Salo et al., 2001). Liotti and co-workers (2000) furthermore showed that different response formats in the Stroop task (verbal, covert, or button press responses) yield differential scalp distributions of the ERPs. Variations in task administration, trial repetition, and related ceiling effects or the type and duration of the investigated meditation practice may have contributed to some diversity in outcomes observed in different studies (Chiesa et al., 2011).

An additional explanation is suggested by new evidence regarding the involvement of the anterior cingulate cortex (ACC) in Stroop-like tasks. The ACC has been shown to be the generator of the LN and to be involved in performance monitoring and response selection (Liotti et al., 2000; Hanslmayr et al., 2008). However, two recent event-related fMRI studies suggest that the role of the ACC is more related to anticipatory regulation of attention rather than the specific selection of responses itself (Roelofs et al., 2006; Aarts et al., 2008). The lack of differential effects in the LN might thus reflect that with extended exposure to the Stroop task anticipatory regulation was perfected in both groups, resulting in the observed ceiling effect. The meditation practice, it seems, has improved earlier stages of processing (indexed by $\mathrm{N} 2$ and P3 changes) that reflect more fundamental changes in attentional processing and are less amenable to simple task repetition effects. Although speculative, this would also explain why 
clear behavioral differences are found when meditators encounter the Stroop tasks for the first time (Chan and Woollacott, 2007; Jha et al., 2007; Moore and Malinowski, 2009), while they tend not to develop on repeated presentation of the same task as was observed here and also reported before (Anderson et al., 2007).

Our results also appear at odds with findings from a longitudinal study carried out by Lutz and co-workers, who found a reduction in RT variability (Lutz et al., 2009) that was not present in out data. There are, however, noteworthy differences to our study in that Lutz et al. investigated changes after much more intensive meditation training (a three-month retreat) and studied the response to rare targets in an auditory task. It might well be that a combination of the already mentioned ceiling effect and the considerable difference in the amount of training accounts for the different outcome.

Despite the lacking evidence of behavioral effects of the meditation practice, significant differences on self-reported mindfulness levels were evident and the increase in mindfulness (FFMQ-total) was correlated with the amount of time participants invested in their meditation practice, suggesting that the time invested in meditation directly translates into recognizable increases in mindfulness. Considering the sample size of $N=$ 12 for this analysis, one needs to be cautious, though, to not over-interpret the results of this correlation.

This study focused on the effects of meditation practice on mechanisms of attentional control as indexed by performance and ERP measures related to the Stroop task. However, we do assume that also other aspects of attention may have been influenced by the meditation practice. A recent paper provides an excellent theoretical account, arguing that mindfulness meditation training, developed over longer periods of time, should lead to the enhancement of cognitive core processes including the sustained monitoring of one's own mental states, the ability to disengage from distracting objects and the skill to redirect attention back to the chosen focus (Slagter et al., 2011). We suggest that the observed changes in the N2 and P3 partially reflect the enhancement of such core processes. In line with this view of more wide-ranging changes, our study also included various other measures, results of which we aim to report elsewhere. These pertain to sustained attention and alertness and the orienting of attention without interfering or conflicting stimuli. In addition, these data will allow us to investigate brain dynamics during rest and meditation practice, where we are particularly interested in global brain states, indexed by oscillating neural activity. Several recent studies suggest that there might be differences between meditators and non-meditators (e.g., Lutz et al., 2004; Tei et al., 2009; Cahn et al., 2010) and between different types of meditation (Travis and Shear, 2010) in this respect. Although not directly related to the methodological approach we were using, it is also worth noting that several studies comparing meditators and non-meditators found differences in brain structure (cortical thickness or gray matter), often in brain areas involved in attentional functions (Lazar et al., 2005; Hölzel et al., 2008; Luders et al., 2009; Grant et al., 2010) and first longitudinal studies show such structural changes in gray and white matter even after relatively brief periods of meditation practice (Tang et al., 2010; Hölzel et al., 2011).

\section{CONCLUSION}

This study adds to the growing body of research indicating the positive effects of meditation training on the neural systems involved in attentional processes. It is one of only a few studies that investigate such changes in a longitudinal fashion and makes several unique contributions. First of all, we showed that a relatively low dose of only $10 \mathrm{~min}$ of practice per day, employed over the course of 16-weeks, significantly changes underlying brain processes that are related to the processing of conflicting stimulus material.

Carrying out a simple mindful breathing meditation for an average of only $10 \mathrm{~min}$ per day for a period of 16-weeks improved neural functioning that is indicative of enhanced focused attentional processing (N2) and less resource intensive object recognition processes (P3), suggesting improvements of neural processing related to attentional core processes. These improvements seem to generalize from the specific situation of a meditation exercise (i.e., focusing on breathing related sensations and maintaining a non-responsive attitude to all arising experiences) to the processing of visually presented stimuli and to the disambiguation of conflicting information present in the stimuli. Based on such generalizations we may speculate that meditation practice addresses very fundamental processes of selective and executive attention that may exhibit its beneficial effects in a variety of domains and situations.

The lack of meditation-specific improvements in behavioral performance may be a result of a too low dose of meditation practice, as several studies with experienced meditators show clearly superior performance. An alternative explanation is that the repeated administration of the same task resulted in performance optimization for all participants, beyond which also the meditators were not able to improve. As the present study cannot distinguish between these explanations, it is advisable to choose the tasks of future longitudinal studies carefully and to limit trial repetition as much as possible, in order to avoid possible ceiling effects.

In sum, these findings provide a positive message to everybody who considers taking up mindfulness meditation practice. Even short, regular meditation practice may hone our attentional systems in a useful fashion.

At the end of the study one of the participants expressed how employing the meditation regime influenced their work performance: "I am completing routine reports in a shorter time period. Also whilst undertaking new tasks I feel that I have a better grasp of understanding complex issues due to improved attention and concentration. (...) It has opened up my train of thought and has led me to think outside the box." Such subjective accounts highlight the relevance of the meditation training beyond the laboratory situation and indicate beneficial effects of meditation practices that do not require any life style changes, some of which we aim to capture by employing cognitive neuroscience methodologies.

\section{ACKNOWLEDGMENTS}

This study was funded by a BIAL Foundation grant (Research Bursary No. 30/08) to Peter Malinowski. 


\section{REFERENCES}

Aarts, E., Roelofs, A., and van Turennout, M. (2008). Anticipatory activity in anterior cingulate cortex can be independent of conflict and error likelihood. J. Neurosci. 28, 4671-4678.

Adorni, R., and Proverbio, A. M. (2009). New insights into name category-related effects: is the age of acquisition a possible factor? Behav. Brain Funct. 5, 33.

Akhtar, N., and Enns, J. T. (1989). Relations between covert orienting and filtering in the development of visual attention. J. Exp. Child Psychol. 48, 315-334.

Anderson, N. D., Lau, M. A., Segal, Z. V., and Bishop, S. R. (2007). Mindfulness-based stress reduction and attentional control. Clin. Psychol. Psychother. 14, 449-463.

Baer, R. A., Smith, G. T., Hopkins, J., Krietemeyer, J., and Toney, L. (2006). Using self-report assessment methods to explore facets of mindfulness. Assessment 13, 27-45.

Baer, R. A., Smith, G. T., Lykins, E., Button, D., Krietemeyer, J., Sauer, S., Walsh, E., Duggan, D., and Williams, J. M. (2008). Construct validity of the five facet mindfulness questionnaire in meditating and nonmeditating samples. Assessment 15, 329-342.

Bell, A. J., and Sejnowski, T. J. (1995). An information-maximization approach to blind separation and blind deconvolution. Neural Comput. 7, 1129-1159.

Benjamini, Y., and Hochberg, Y. (1995). Controlling the false discovery rate: a practical and powerful approach to multiple testing. J. R. Stat. Soc. Ser. B 57, 289-300.

Bhikkhu Bodhi (1995). The Middle Length Discourses of the Buddha. Boston, MA: Wisdom Publications.

Bishop, S. R. (2002). What do we really know about mindfulnessbased stress reduction? Psychosom. Med. 64, 71-84.

Bishop, S. R., Lau, M. A., Shapiro, S. L., Carlson, L. E., Anderson, N. D., Carmody, J., Segal, Z. V., Abbey, S., Speca, M., Velting, D., and Devins, G. (2004). Mindfulness: a proposed operational definition. Clin. Psychol. Sci. Pract. 11, 230-242.

Bosch-Bayard, J., Valdés-Sosa, P., Virués-Alba, E., Aubert-Vázquez, E., John, R., Harmony, T., RieraDíaz, J., and Trujillo-Barreto, N. (2001). 3D statistical parametric mapping of variable resolution electromagnetic tomography (VARETA). Clin. Electroencephalogr. $32,47-66$.
Brefczynski-Lewis, J. A., Lutz, A., Schaefer, H. S., Levinson, D. B., and Davidson, R. J. (2007). Neural correlates of attentional expertise in long-term meditation practitioners. Proc. Natl. Acad. Sci. U.S.A. 104, 11483-11488.

Cahn, B. R., Delorme, A., and Polich, J. (2010). Occipital gamma activation during Vipassana meditation. $\operatorname{Cog} n$. Process. 11, 39-56.

Cahn, B. R., and Polich, J. (2006) Meditation states and traits: EEG, ERP, and neuroimaging studies. Psychol. Bull. 132, 180-211.

Cahn, B. R., and Polich, J. (2009). Meditation (Vipassana) and the P3a event-related brain potential. Int. J. Psychophysiol. 72, 51-60.

Chambers, R., Gullone, E., and Allen, N. B. (2009). Mindful emotion regulation: an integrative review. Clin. Psychol. Rev. 29, 560-572.

Chambers, R., Lo, B. C. Y., and Allen, N. B. (2008). The impact of intensive mindfulness training on attentional control, cognitive style, and affect. Cognit. Ther. Res. 32, 303-322.

Chan, D., and Woollacott, M. (2007) Effects of level of meditation experience on attentional focus: is the efficiency of executive or orientation networks improved? J. Altern. Complement. Med. 13, 651-657.

Chiesa, A., Calati, R., and Serretti, A. (2011). Does mindfulness training improve cognitive abilities? A systematic review of neuropsychological findings. Clin. Psychol. Rev. 31, 449-464.

Chiesa, A., and Malinowski, P. (2011). Mindfulness based interventions: are they all the same? J. Clin. Psychol. 67, 404-424.

Chiesa, A., and Serretti, A. (2009). Mindfulness-based stress reduction for stress management in healthy people: a review and meta-analysis. J. Altern. Complement. Med. 15, 593-600.

Chiesa, A., and Serretti, A. (2011). Mindfulness based cognitive therapy for psychiatric disorders: a systematic review and meta-analysis. Psychiatry Res. 187, 441-453.

Christie, J., and Klein, R. (1995) Familiarity and attention: does what we know affect what we notice? Mem. Cognit. 23, 547-550.

Cohen, L., and Dehaene, S. (2004). Specialization within the ventral stream: the case for the visual word form area. Neuroimage 22, 466-476.

Cohen, L., Lehericy, S., Chochon, F., Lemer, C., Rivaud, S., and Dehaene, S. (2002). Language-specific tuning of visual cortex? Functional properties of the Visual Word Form Area. Brain 125, 1054-1069.

Corbetta, M., and Shulman, G. L. (2002). Control of goal-directed and stimulus-driven attention in the brain. Nat. Rev. Neurosci. 3, 201-215.

Delorme, A., and Makeig, S. (2004). EEGLAB: an open source toolbox for analysis of single-trial EEG dynamics including independent component analysis. J. Neurosci. Methods 134, 9-21.

Dorjee, D. (2010). Kinds and dimensions of mindfulness: why it is important to distinguish them. Mindfulness 1, 152-160.

Eimer, M. (1997). An event-related potential (ERP) study of transient and sustained visual attention to color and form. Biol. Psychol. 44, 143-160.

Evans, A. C., Collins, D. L., Mills, S R., Brown, E. D., Kelly, R. L., and Peters, T. M. (1993). “3D statistical neuroanatomical models from 305 MRI volumes," in Nuclear Science Symposium and Medical Imaging Conference, 1993 IEEE Conference Record, 1813-1817.

Folstein, J. R., and van Petten, C. (2008). Influence of cognitive control and mismatch on the N2 component of the ERP: a review. Psychophysiology 45, 152-170.

Grant, J. A., Courtemanche, J. Duerden, E. G., Duncan, G. H. and Rainville, P. (2010). Cortical thickness and pain sensitivity in zen meditators. Emotion 10, 43-53.

Green, C. S., and Bavelier, D. (2008) Exercising your brain: a review of human brain plasticity and training-induced learning. Psychol. Aging 23, 692-701.

Grossman, P., Niemann, L., Schmidt, S., and Walach, H. (2004). Mindfulness-based stress reduction and health benefits. A meta-analysis. J. Psychosom. Res. 57, 35-43.

Gruber, T., Trujillo-Barreto, N. J. Giabbiconi, C. M., Valdes-Sosa, P. A., and Müller, M. M. (2006). Brain electrical tomography (BET) analysis of induced gamma band responses during a simple object recognition task. Neuroimage 29, 888-900.

Gunaratana, H. (1992). Mindfulness in Plain English. Boston, MA: Wisdom Publications.

Hanslmayr, S., Pastotter, B., Bauml, K. H., Gruber, S., Wimber, M., and Klimesch, W. (2008). The electrophysiological dynamics of interference during the Stroop task. J. Cogn. Neurosci. 20, 215-225.
Hillyard, S. A., and Anllo-Vento, L. (1998). Event-related brain potentials in the study of visual selective attention. Proc. Natl. Acad. Sci. U.S.A. 95, 781-787.

Hillyard, S. A., Vogel, E. K., and Luck, S. J. (1998). Sensory gain control (amplification) as a mechanism of selective attention: electrophysiological and neuroimaging evidence. Philos. Trans. R. Soc. Lond. B Biol. Sci. 353, 1257-1270.

Hodgins, H. S., and Adair, K. C. (2010). Attentional processes and meditation. Conscious. Cogn. 19, 872-878.

Hölzel, B. K., Carmody, J., Vangel, M., Congleton, C., Yerramsetti, S. M., Gard, T., and Lazar, S. W. (2011). Mindfulness practice leads to increases in regional brain gray matter density. Psychiatry Res. 191, 36-43.

Hölzel, B. K., Ott, U., Gard, T., Hempel, H., Weygandt, M., Morgen, K., and Vaitl, D. (2008). Investigation of mindfulness meditation practitioners with voxel-based morphometry. Soc. Cogn. Affect. Neurosci. 3, 55-61.

Ilan, A. B., and Polich, J. (1999). P300 and response time from a manual Stroop task. Clin. Neurophysiol. 110, 367-373.

Jacobs, T. L., Epel, E. S., Lin, J., Blackburn, E. H., Wolkowitz, O. M., Bridwell, D. A., Zanesco, A P., Aichele, S. R., Sahdra, B. K., Maclean, K. A., King, B. G., Shaver, P. R., Rosenberg, E. L., Ferrer E., Wallace, B. A., and Saron, C. D. (2011). Intensive meditation training, immune cell telomerase activity, and psychological mediators. Psychoneuroendocrinology 36, 664-681.

Jha, A. P., Krompinger, J., and Baime, M. J. (2007). Mindfulness training modifies subsystems of attention. Cogn. Affect. Behav. Neurosci. 7, 109-119.

Kabat-Zinn, J. (1990). Full Catastrophe Living: Using the Wisdom of Your Body and Mind to Face Stress, Pain and Illness. New York, NY: Bantam Doubleday Dell Publishing.

Kabat-Zinn, J. (2003). Mindfulnessbased interventions in context: past, present, and future. Clin. Psychol. Sci. Pract. 10, 144-156.

Kabat-Zinn, J., Lipworth, L., and Burney, R. (1985). The clinical use of mindfulness meditation for the self-regulation of chronic pain J. Behav. Med. 8, 163-190.

Kabat-Zinn, J., Lipworth, L., Burney, R., and Sellers, W. (1987). Four-year follow-up of a meditation-based 
program for the self-regulation of chronic pain: treatment outcomes and compliance. Clin. J. Pain 2, 159-173.

Kabat-Zinn, J., Massion, A. O., Kristeller, J., Peterson, L. G., Fletcher, K. E., Pbert, L., Lenderking, W. R., and Santorelli, S. F. (1992). Effectiveness of a meditation-based stress reduction program in the treatment of anxiety disorders. Am. J. Psychiatry 149, 936-943.

Karmapa Wangchug Dorje (2009). Mahamudra - The Ocean of True Meaning: The Profound Instructions on Coexisting Unity, the Essence of the Ocean of True Meaning, and Light Radiating Activity. Münster: Edition Octopus.

Kindt, M., Bierman, D., and Brosschot, J. F. (1996). Stroop versus Stroop: comparison of a card format and a single-trial format of the standard color-word Stroop task and the emotional Stroop task. Pers. Individ. Dif. 21, 653-661.

Kozasa, E. H., Sato, J. R., Lacerda, S. S., Barreiros, M. A., Radvany, J., Russell, T. A., Sanches, L. G., Mello, L. E., and Amaro, E. Jr. (2012). Meditation training increases brain efficiency in an attention task. Neuroimage 59, 745-749.

Lazar, S. W., Kerr, C. E., Wasserman, R. H., Gray, J. R., Greve, D. N., Treadway, M. T., Mcgarvey, M., Quinn, B. T., Dusek, J. A., Benson, H., Rauch, S. L., Moore, C. I., and Fischl, B. (2005). Meditation experience is associated with increased cortical thickness. Neuroreport 16, 1893-1897.

Liotti, M., Woldorff, M. G., Perez, R., and Mayberg, H. S. (2000). An ERP study of the temporal course of the Stroop color-word interference effect. Neuropsychologia 38, 701-711.

Luders, E., Toga, A. W., Lepore, N., and Gaser, C. (2009). The underlying anatomical correlates of longterm meditation: larger hippocampal and frontal volumes of gray matter. Neuroimage 45, 672-678

Lutz, A., Greischar, L. L., Rawlings, N. B., Ricard, M., and Davidson, R. J. (2004). Long-term meditators self-induce high-amplitude gamma synchrony during mental practice. Proc. Natl. Acad. Sci. U.S.A. 101, 16369-16373.

Lutz, A., Slagter, H. A., Dunne, J. D., and Davidson, R. J. (2008). Attention regulation and monitoring in meditation. Trends Cogn. Sci. $12,163-169$.

Lutz, A., Slagter, H. A., Rawlings, N. B., Francis, A. D., Greischar, L. L., and Davidson, R. J. (2009). Mental training enhances attentional stability: neural and behavioral evidence. J. Neurosci. 29, 13418-13427.

Lyubomirsky, S., and Lepper, H. S. (1999). A measure of subjective happiness: preliminary reliability and construct validation. Soc. Indic. Res. 46, 137-155.

Lyubomirsky, S., and Tucker, K. L. (1998). Implications of individual differences in subjective happiness for perceiving, interpreting, and thinking about life events. Motiv. Emot. 22, 155-186.

MacLean, K. A., Ferrer, E., Aichele, S. R., Bridwell, D. A., Zanesco, A. P. Jacobs, T. L., King, B. G., Rosenberg, E. L., Sahdra, B. K., Shaver, P. R., Wallace, B. A., Mangun, G. R., and Saron, C. D. (2010). Intensive meditation training improves perceptual discrimination and sustained attention. Psychol. Sci. 21 829-839.

MacLeod, C. M. (1991). Half a century of research on the Stroop effect: an integrative review. Psychol. Bull. 109, 163-203.

Malinowski, P. (2008). Mindfulness as psychological dimension: concepts and applications. Ir. J. Psychol. 29, 155-166.

Malinowski, P. (2012). "Flourishing through meditation and mindfulness," in Oxford Handbook of Happiness, eds I. Boniwell and S. David. (Oxford: Oxford University Press).

Miyake, A., Friedman, N. P., Emerson, M. J., Witzki, A. H., Howerter, A. and Wager, T. D. (2000). The unity and diversity of executive functions and their contributions to complex "Frontal Lobe" tasks: a latent variable analysis. Cogn. Psychol. 41, 49-100.

Moore, A., and Malinowski, P. (2009). Meditation, mindfulness and cognitive flexibility. Conscious. Cogn. 18, 176-186.

Nolan, H., Whelan, R., and Reilly, R. B. (2010). FASTER: Fully Automated Statistical Thresholding for EEG artifact Rejection. J. Neurosci. Methods 192, 152-162.

Polak, E. L. (2009). Impact of Two Sessions of Mindfulness Training on Attention. Ph.D. thesis, University of Miami.

Polich, J. (2007). Updating P300: an integrative theory of $\mathrm{P} 3 \mathrm{a}$ and P3b. Clin. Neurophysiol. 118, 2128-2148.

Posner, M. I., and Rothbart, M. K. (2007). Research on attention networks as a model for the integration of psychological science. Annu. Rev. Psychol. 58, 1-23.
Raz, A., and Buhle, J. (2006) Typologies of attentional networks. Nat. Rev. Neurosci. 7, 367-379.

Roelofs, A., van Turennout, M., and Coles, M. G. (2006). Anterior cingulate cortex activity can be independent of response conflict in Stroop-like tasks. Proc. Natl. Acad. Sci. U.S.A. 103, 13884-13889.

Sahdra, B. K., Maclean, K. A., Ferrer E., Shaver, P. R., Rosenberg, E. L., Jacobs, T. L., Zanesco, A. P. King, B. G., Aichele, S. R., Bridwell, D. A., Mangun, G. R., Lavy, S. Wallace, B. A., and Saron, C. D. (2011). Enhanced response inhibition during intensive meditation training predicts improvements in self-reported adaptive socioemotional functioning. Emotion 11, 299-312.

Salo, R., Henik, A., and Robertson, L. C. (2001). Interpreting Stroop interference: an analysis of differences between task versions. Neuropsychology 15, 462-471.

Schendan, H. E., and Kutas, M (2002). Neurophysiological evidence for two processing times for visual object identification. Neuropsychologia 40, 931-945.

Schendan, H. E., and Stern, C. E. (2007). Mental rotation and object categorization share a common network of prefrontal and dorsal and ventral regions of posterior cortex. Neuroimage 35, 1264-1277.

Segal, Z., Williams, J. M. G., and Teasdale, J. D. (2002). Mindfulnessbased Cognitive Therapy for Depression: A New Approach for Preventing Relapse. New York, NY: Guilford.

Semple, R. J. (2010). Does mindfulness meditation enhance attention? A randomized controlled trial. Mindfulness 1, 121-130.

Shapiro, K. L., Arnell, K. M., and Raymond, J. E. (1997). The attentional blink. Trends Cogn. Sci. 1, 219-296.

Shapiro, S. L., Carlson, L. E., Astin, J. A., and Freedman, B. (2006). Mechanisms of mindfulness. J. Clin. Psychol. 62, 373-386.

Shaywitz, B. A., Shaywitz, S. E. Blachman, B. A., Pugh, K. R., Fulbright, R. K., Skudlarski, P., Mencl, W. E., Constable, R. T. Holahan, J. M., Marchione, K. E. Fletcher, J. M., Lyon, G. R., and Gore, J. C. (2004). Development of left occipitotemporal systems for skilled reading in children after a phonologically- based intervention. Biol. Psychiatry 55, 926-933.

Slagter, H. A., Davidson, R. J., and Lutz, A. (2011). Mental training as a tool in the neuroscientific study of brain and cognitive plasticity. Front. Hum. Neurosci. 5, 17. doi: 10.3389/fnhum.2011.00017

Slagter, H. A., Lutz, A., Greischar, L. L., Francis, A. D., Nieuwenhuis, S., Davis, J. M., and Davidson, R. J. (2007). Mental training affects distribution of limited brain resources. PLoS Biol. 5, e138. doi: 10.1371/journal.pbio.0050138

Slagter, H. A., Lutz, A., Greischar, L. L., Nieuwenhuis, S., and Davidson, R. J. (2009). Theta phase synchrony and conscious target perception: impact of intensive mental training. J. Cogn. Neurosci. 21, 1536-1549.

Stroop, J. R. (1935). Studies of interference in serial verbal reactions. J. Exp. Psychol. 18, 643-661.

Tang, Y. Y., Lu, Q., Geng, X., Stein, E. A., Yang, Y., and Posner, M. I. (2010). Short-term meditation induces white matter changes in the anterior cingulate. Proc. Natl. Acad. Sci. U.S.A. 107, 15649-15652.

Tang, Y. Y., Ma, Y., Fan, Y., Feng, H., Wang, J., Feng, S., Lu, Q., Hu, B., Lin, Y., Li, J., Zhang, Y., Wang, Y., Zhou, L., and Fan, M. (2009). Central and autonomic nervous system interaction is altered by shortterm meditation. Proc. Natl. Acad. Sci. U.S.A. 106, 8865-8870.

Tei, S., Faber, P. L., Lehmann, D. Tsujiuchi, T., Kumano, H., PascualMarqui, R. D., Gianotti, L. R., and Kochi, K. (2009). Meditators and non-meditators: EEG source imaging during resting. Brain Topogr. 22, 158-165.

Travis, F., and Shear, J. (2010). Focused attention, open monitoring and automatic self-transcending: Categories to organize meditations from Vedic, Buddhist and Chinese traditions. Conscious. Cogn. 19, 1110-1118.

Valentine, E. R., and Sweet, P. L. G. (1999). Meditation and attention: comparison of the effects of concentrative and mindfulness meditation on sustained attention. Ment. Health Relig. Cult. 2, 59-70.

van den Hurk, P. A., Giommi, F., Gielen, S. C., Speckens, A. E., and Barendregt, H. P. (2010). Greater efficiency in attentional processing related to mindfulness meditation. Q. J. Exp. Psychol. 63, 1168-1180

van Leeuwen, S., Müller, N. G., and Melloni, L. (2009). Age effects on attentional blink performance in meditation. Conscious. Cogn. 18, 593-599.

van Vugt, M. K., and Jha, A. P. (2011). Investigating the impact of mindfulness meditation training 
on working memory: a mathematical modeling approach. Cogn. Affect. Behav. Neurosci. 11, 344-353.

Wenk-Sormaz, H. (2005). Meditation can reduce habitual responding. Altern. Ther. Health Med. 11, 42-58.

Williams, J. M. (2010). Mindfulness and psychological process. Emotion $10,1-7$.
Zeidan, F., Johnson, S. K., Diamond, B. J., David, Z., and Goolkasian, P. (2010). Mindfulness meditation improves cognition: evidence of brief mental training. Conscious. Cogn. 19, 597-605.

Conflict of Interest Statement: The authors declare that the research was conducted in the absence of any commercial or financial relationships that could be construed as a potential conflict of interest.

Received: 31 October 2011; accepted: 29 January 2012; published online: 10 February 2012.

Citation: Moore A, Gruber T, Derose $J$ and Malinowski P (2012) Regular, brief mindfulness meditation practice improves electrophysiological markers of attentional control. Front. Hum.
Neurosci. 6:18. doi: 10.3389/fnhum. 2012.00018

Copyright (C) 2012 Moore, Gruber, Derose and Malinowski. This is an openaccess article distributed under the terms of the Creative Commons Attribution Non Commercial License, which permits non-commercial use, distribution, and reproduction in other forums, provided the original authors and source are credited. 